\title{
How post-processing by selective thermal reflow can reduce the roughness of 3D lithography in micro-optical lenses
}

\author{
Robert Kirchner ${ }^{1}$, Nachiappan Chidambaram ${ }^{1}$, Mirco Altana ${ }^{2}$, Helmut Schift ${ }^{1, *}$ \\ ${ }^{1}$ Paul Scherrer Institute, Laboratory for Micro and Nanotechnology, 5232 Villigen PSI, Switzerland \\ ${ }^{2}$ Heptagon Oy, Moosstrasse 2, 8803 Rüschlikon, Switzerland
}

\begin{abstract}
Most polymeric refractive micro-optics simultaneously demand ultra-smooth 3D topographies and precise geometry for high optical performance and low stray light. We have established a surface selective smoothening for thermoplastic polymers that does not affect the designed optical geometry. For example, high curvature corners required for a $50 \mu \mathrm{m}$ tall optical diffuser device were maintained while the surface roughness was reduced to about $10 \mathrm{~nm}$ RMS. 3D master structures were fabricated using direct write laser-lithography with two-photon absorption. Master structures were then replicated into poly(methyl methacrylate) through a poly(dimethyl siloxane) intermediate copying step and subsequently smoothed-out by high-energy photon exposure and thermal post-processing. The smoothening results in a lower roughness compared to a direct writing strategy using even about $50 \mathrm{~nm}$ vertical discretization steps still enables 10 times faster writing times.
\end{abstract}

Keywords: Micro-lens array, glass transition temperature, surface roughness, polymer, 2-photon polymerization

\section{INTRODUCTION}

This work deals with a new method that enables the fabrication of micro-lenses with ultra-smooth, optical grade surfaces. Instead of improving surface roughness by time-consuming and sometime design-specific direct-writing strategies of novel 3D lithographic methods, we chose a post-processing method based on surface selective thermal reflow. We will present a method for modification of polymer surfaces and compare the two strategies post-processing and direct-writing for surface smoothening [1].

There are novel fabrication schemes including 3D lithography methods such as grayscale lithography and 2-photon polymerization (2PP) lithography that build the lenses out of thin layers. Grayscale approaches are either based on masks with varying transmission grades (grayscale masks) or direct writing methods [2-5]. While the first one is well established for i-line lithography (365 nm wavelength), higher resolution methods are based on sequential and scanned photon, electron beam or ion beam writing. Direct laser writing (DLW) lithography with a lateral resolution of about 700 $\mathrm{nm}$ is well suited for refractive micro-optics, its vertical constraint to resist films below $30 \mu \mathrm{m}$ limits its use for freeform and complex designs beyond this thickness. This is mainly due to the fact that the entire film is exposed along the beam when using single photon absorption processes. Similarly, the higher resolution grayscale methods based on electron and ion beams are mostly suitable for diffractive optics with film resist thickness below $10 \mu \mathrm{m}$ for electrons and even thinner for ions [6,7]. A true 3D capable method such as 2PP-DLW allows for full freeform designs without restrictions on the size of the micro-optics [8]. Exemplary realized designs are micro-prisms [9], single-tier micro-lenses [10,11] and multitier lenses [12]. As mentioned above, the typical limitation of 2PP is the writing time and thus writing area and height. This not only because of the high resolution of the beam in lateral direction, but also the additional confinement of the polymerizing laser beam in vertical direction, which makes fully 3D structures possible but also requires extensive layering for more bulky structures. The laser beam focus (a spheroid called voxel), is scanned through a viscous material and polymerizes the material on its track, which means that the structure is built from trajectories which are assembled like woodpiles.

*helmut.schift@psi.ch; phone +41 56310 2839; fax +41 56310 2646; www.psi.ch/lmn 


\section{CONSIDERING ROUGHNESS IN MICRO-OPTICS}

\subsection{Roughness of micro-optical lenses}

Good imaging optics require an overall surface roughness about $1 / 100^{\text {th }}$ of the wavelength used, which results in low stray light, high resolution and good imaging contrast. For visible wavelengths $(400-780 \mathrm{~nm})$, this would be a roughness $\mathrm{R}_{\mathrm{q}}$ (geometric average or root mean square roughness RMS) of below 4-8 $\mathrm{nm}$. The same applies for refractive micro-optical elements, with diameters from a few micrometers up to a few millimeters. They are nowadays used in different applications, e.g., as imaging element on miniaturized optics such as for a camera, optical sensors, diffusors (e.g., for flashes) or for movement tracking (in smartphones, computers, computer game consoles, and surveillance sensors). Since these components are highly integrated and have a small footprint, they have to be fabricated by novel 3D lithographic methods [2,3,8]. Furthermore they are typically replicated by plastic molding techniques in large quantities such as by hot embossing, thermal injection molding, casting or UV-assisted methods. Typical industrial relevant thermoplastic polymers, are commodity plastics such as poly(carbonate) (PC), poly(methyl methacrylate) (PMMA) or other thermoplastic polymers with appropriate thermo-mechanical, optical and/or chemical properties. While glass polishing for macroscopic lenses easily achieves roughness of below $2 \mathrm{~nm}$, replicated lenses require already molds with the same surface roughness as the final product.

The milling methods typically employed for the patterning of metal molds are often well above this roughness value, but can be improved to meet these requirements. Although, according to Ref. [13], single point diamond turned surfaces (e.g., in brass) can achieve $\mathrm{R}_{\mathrm{q}}$ of $5 \mathrm{~nm}$ and sometimes even down to $3 \mathrm{~nm}$, this is far from being standard. The Society for the Plastics Industry (SPI) has a range of specifications for the finish of molds for plastic, and A-1 is the smoothest. It calls for $\mathrm{R}_{\mathrm{a}}$ (arithmetic average roughness) between 0.5 and 1.0 micro-inch $(12.5-25.0 \mathrm{~nm})$. Given that the measure is $\mathrm{R}_{\mathrm{a}}$ (about $0.7 \cdot \mathrm{R}_{\mathrm{q}}$ ) and the surface could be as rough as $25 \mathrm{~nm}\left(35 \mathrm{~nm} \mathrm{R} \mathrm{R}_{\mathrm{q}}\right.$ ), and considering the $1 / 100^{\text {th }}$ rule mentioned above, this is a very loose tolerance for optical surfaces, and normally this cannot be accepted. An empirical expression relating reflection efficiency of a conductive mirror with roughness and wavelength was derived to be [14]:

$$
\frac{R_{q}}{R_{0}}=e^{-\left(\frac{4 \pi R_{q}}{\lambda}\right)^{2}}
$$

or, for inclined incidence with angle $\alpha$ :

$$
\frac{R_{q}}{R_{0}}=e^{-\left(\frac{4 \pi R_{q} \cos \alpha}{\lambda}\right)^{2}}
$$

where $R_{s}$ is the specular reflectance of the surface and $R_{0}$ the specular reflectance of an ideal surface with no roughness and very high conductivity. This relation imposes very strong restrictions on the roughness.

Because many replication methods can achieve faithful replication of sub-10 nm surface topographies, a fabrication method for a structure intended as a mold for replication would need the first original to be as smooth as possible. However, very often the pattern methods entail a trade-off between processing time and the resolution to be achieved. With current 3D lithographic methods, direct fabrication of the required ultra-smooth surfaces can lead to substantial increase of writing times which are not supportable for many large area applications. In this publication we present ways to reduce roughness in micro-optical elements. The application area will be micro-optics, but the process is general enough that it can be adapted to other micro- or even macro-elements which need surface finish within the range described above.

\subsection{Layering versus post-processing for roughness reduction}

There are three main possibilities to minimize the roughness in 2PP (Figure 1): (a) voxel-based methods such as increasing the voxel overlap by having a fine vertical/horizontal discretization or reducing the voxel size by varying the polymerization threshold/dose, (b) writing strategies such as varying the vertical discretization based on the curvature of the feature to be written or writing curved out-of-plane instead of flat in-plane trajectories, (c) post processing methods such as the one described in this work. Increasing the overlap reduces the roughness to some extent [15], but resist tends 
toward micro-explosions for a higher overlap due to the dielectric breakdown of the polymerized material [16]. Reducing the voxel size has only small process latitude. With variable vertical discretization, roughness can be reduced [17], but one needs to dynamically tune the dose to avoid micro-explosions. Out-of-plane trajectories can give some improvements [18] but this is essentially shifting the roughness problem from ' $z$ ' axis to ' $x$ or $y$ ' axis. Furthermore, high resolution features and high aspect ratio structures, will suffer the most from writing strategy approaches that will remain reserved for special geometries [12]. We believe a universal post processing is the appropriate strategy for attaining a super smooth surface while having limited change in the surface profile alongside acceptable writing times.

\section{Voxel size}

- Laser dose / threshold

- Exposure optics and resist with «diffusive» component
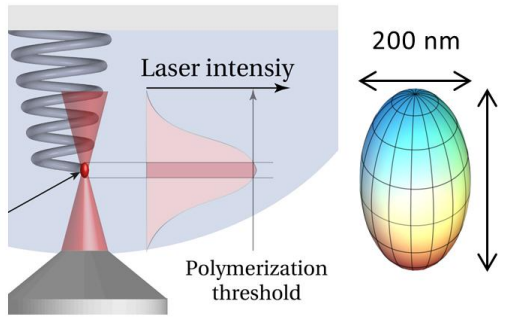

\section{Writing strategies}

- Increasing the voxel overlap

- Variable height slicing

- Arc scanning

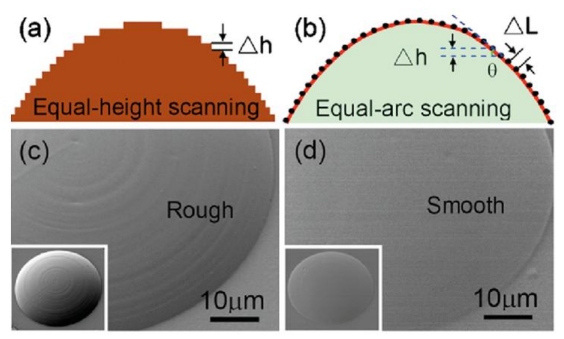

\section{Post processing}

- «Reflow» of resist

- Replication required

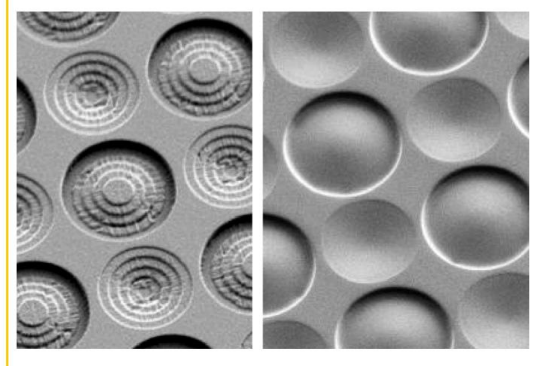

Figure 1. Comparison of three main alternatives for surface smoothening either by (a) variation of voxel size (with exposure dose / resist chemistry) or (b) by writing strategies or (c) by post-processing. Modified and reproduced by permission. @ 2010 AIP.

While piezo drives of current 2PP-DLW systems allow for the scanning of trajectories in all directions, fast writing is often achieved when the in-plane scanning mechanism in lateral direction is done via fast galvanic scanners. This allows layer-by-layer writing for which particularly the layering in vertical direction becomes the time-limiting factor of the final 3D design. The high resolution focal spot size between about $200 \mathrm{~nm}$ in lateral and $600 \mathrm{~nm}$ in vertical direction gives depending on the layer-to-layer increment as well as the in-plane rastering a certain surface roughness in the range of focal spot size. In general, a finer rastering and design discretization allows for a smoother surface while it directly impacts the total writing effort and time. E.g., the writing time would scale linearly in each direction, and thus an increase of overlap from zero to $50 \%$ of adjacent layers in only one direction would increase the exposure time by a factor of 1.5 , and an overlap of $90 \%$ would multiply it by a factor of 11 .

Instead of the increase of overlap, we chose a post-processing method which allows writing the micro-optical lenses with high speed, but large increments, and added a surface smoothening ("polishing") process after the writing (Figure 2). Since the process needs to be based on non-contact and should not involve a coating step, it requires a surface selective modification of the polymer in a depth which is comparable to the surface roughness we want to smoothen out. Such surface selective modification is possible by exposure of the polymer with intense radiation or high energy beams of electrons and ions (see Figure 2). Similarly to the process established for grayscale electron beam lithography structures, the thermally activated selective topography equilibration (TASTE) process, we established a reduction of the molecular weight of the polymer and thus the glass transition temperature with a high degree of localized vertical confinement [19].

The available methods do not only differ in penetration depth, but due to their ways of interaction with the polymer surface also in surface ablation. For the purpose of surface smoothening, vacuum UV-light (VUV) with $172 \mathrm{~nm}$ wavelength provided the best capabilities. It can be operated in ambient, and large area lamps enabling homogeneous irradiation are available. More details will be presented in future publications [20]. 


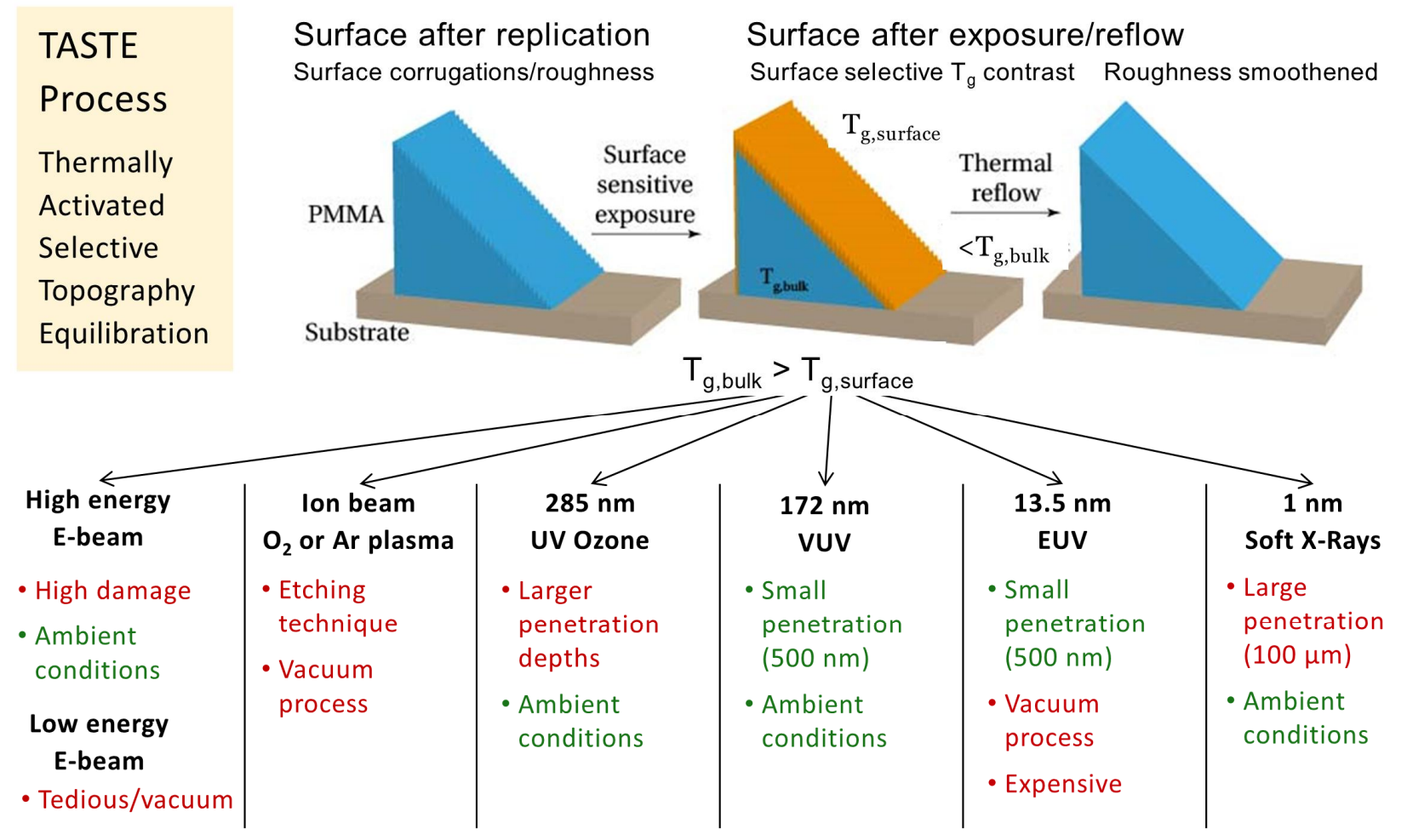

Figure 2. Different process possibilities for generating surface sensitive damage which can be used for thermally activated selective topography equilibration (TASTE): Exposure with low energy e-beam, $172 \mathrm{~nm}$ wavelength VUV and $13.5 \mathrm{~nm}$ wavelength EUV are known to enable permanent modification in a skin layer of less than $500 \mathrm{~nm}$ only, with low ablation and roughening in contrast to plasma treatment. VUV is available in commercial tools for large area exposure in ambient.

\section{PROCESS ROUTE FOR GENERATION OF REPLICATED MICRO-LENSES}

Since the TASTE process is best explored with linear thermoplastic polymers such as PMMA, but did not yet show good results with negative resists, we chose two replication steps which allowed transferring the lenses from the crosslinked proprietary material optimized for 2PP-DLW (IpDip and IpS from NanoScribe GmbH) to PMMA (Figure 3). For this the lens structures were written with the Nanoscribe Photonic GT in IpDip with moderate scan speeds provided by the galvanic scanner $(20 \mathrm{~mm} / \mathrm{sec}$ ) and low pulse intensity (around $1 \mathrm{~nJ}$ ), and using $500 \mathrm{~nm}$ slicing distance between adjacent vertical layers. This resulted in an in-plane roughness of about $20 \mathrm{~nm}$ RMS and respective steps due to the coarse discretization. For comparison, the same structures were exposed in IpS, a resist optimized for higher throughput and lower surface roughness, with different slicing distances. The process chain for the upscaling of lens fabrication involves a further copying of lens structures by molding techniques. We chose a method of bulk filling instead of writing a scaffold with reduced overlap, resulting in hollow structures which would be more prone to collapse and excessive shrinkage.

The voxel size using a $63 \times$ NA 1.4 microscope objective for the IpDip is typically about $200 \times 200 \times 600 \mathrm{~nm}^{3}$ while for the IpS it is enlarged to $300 \times 300 \times 900 \mathrm{~nm}^{3}$ and for the $25 \mathrm{x}$ NA 0.8 objective we anticipate a voxel size of about $700 \times 700 \times 2100 \mathrm{~nm}^{3}$, including the blurring due to the chemical nature of the resist. Thus the overlap of first IpDip structure is about $100(17 \%)$ for the $63 \mathrm{x}$ objective while the overlap for the $\mathrm{IpS}$ was varied from $1600 \mathrm{~nm}(76 \%, 500 \mathrm{~nm}$ slicing distance) to $2050 \mathrm{~nm}(98 \%, 50 \mathrm{~nm}$ slicing distance). The writing time for a $3 \times 3$ microlens array (MLA) was about $5 \mathrm{~min}$ for $500 \mathrm{~nm}$ slicing and $50 \mathrm{~min}$ for $50 \mathrm{~nm}$ slicing. This translates into a writing time for a MLA device with about $2.4 \mathrm{~mm}$ diameter of about $48 \mathrm{~h}$ or $470 \mathrm{~h}$, respectively.

For structures used for surface smoothening by post-processing, the IpDip original was first copied into a UV-curing PDMS (micro resist technology $\mathrm{GmbH}$ ), as alternative to OrmoStamp (micro resist technology $\mathrm{GmbH}$ ), which is flexible 
but at the same time hard enough that both the structures and the roughness can be replicated without loss, and then by thermal imprint into PMMA120k (micro resist technology $\mathrm{GmbH}$ ). More details on the replication process can be found in Refs. [10,21-24].

The PMMA patterns were exposed for $30 \mathrm{~s}$ with a VUV intensity of about $16.3 \mathrm{~mW} / \mathrm{cm}^{2}$ at $172 \mathrm{~nm}$ wavelength in a flood exposure tool (EX-mini, Hamamatsu Corp.). After exposure, the lenses were annealed in a top-heater setup at a temperature of $140^{\circ} \mathrm{C}$ for $15 \mathrm{~min}$. This setup allows for generating a temperature gradient from the top of the lenses towards the base which additionally supports the concept of surface limited softening. After reflow, the samples were immediately cooled to room temperature, coated with 5-10 nm chromium and inspected in scanning electron microscopy (SEM, Zeiss Supra 55 VP, Zeiss Microscopy).

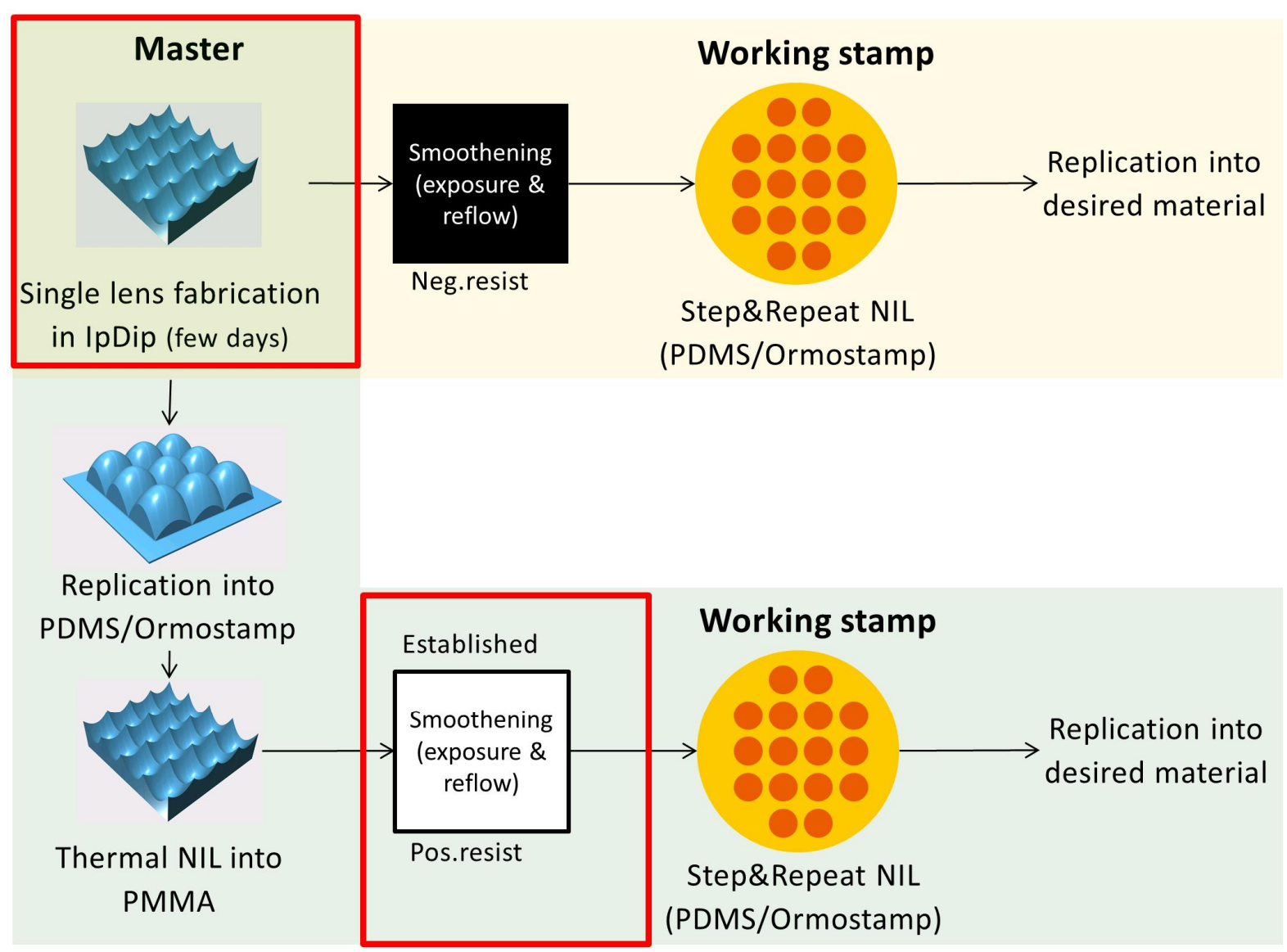

Figure 3. Principal process routes for replication of micro-lenses, fabricated by two-photon direct write laser-lithography (2PP-DLW) in negative tone resist (IpDip). Only the copying into a thermoplastic material enables the use of the TASTE process in a post-exposure thermal reflow. The optical elements with smoothened surface can then be used for further upscaling into a working tool by step\&repeat imprint. Red frames indicate the initial and the reflowed lens. 


\section{RESULTS AND COMPARISON OF METHODS}

The IpDip master pattern was faithfully replicated into PMMA including the step-to-step roughness due to the writing trajectories used for model discretization (Figure 4). The PMMA replication resulted in a relatively thick residual layer which supported together with the flexible PDMS mold a defect free release of the microstructures, the high-aspect ratio features as well as the nanotexture on the lens surface. As found in a previous study [10], the PDMS allowed for a very broad process window $\left(0.5-5 \mathrm{MPa}\right.$ imprint pressure, $140-220^{\circ} \mathrm{C}$ imprint temperature, $1 \mathrm{~s}-180 \mathrm{~min}$ pressure holding time) which makes the replication process very appealing as an industrial process.

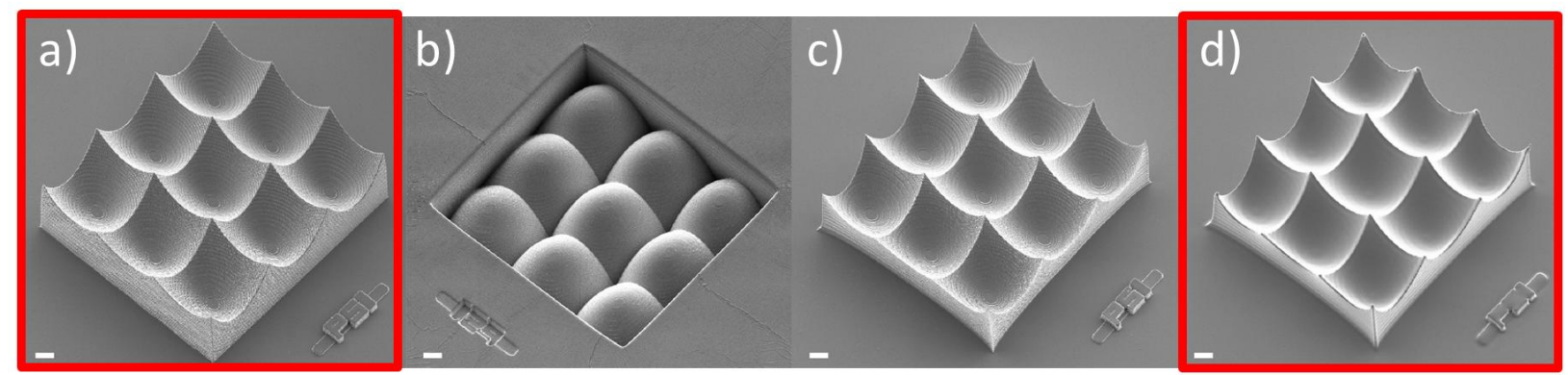

Figure 4. Process steps from a) original after 2PP-DLW in IpDip (63x objective, slicing distance $500 \mathrm{~nm})$, b) replication by UV-casting into UV-PDMS, c) thermal NIL into PMMA, d) surface smoothening by exposure and thermal reflow. Red frames indicate the initial and the reflowed lens. Scale bar $10 \mu \mathrm{m}$. Modified and reproduced by permission. @ 2016 AVS

Figure 5 displays SEM micrographs of a $3 \times 3$ microlens array manufactured by 2 PP-DLW displaying the effect of variation of voxel size, writing strategy and post-processing on surface roughness. We choose the $63 \mathrm{x}$ objective lens for the IpDip resist which was optimized for highest resolution, but also exhibits the most prominent roughness due to the small voxel size. For comparison, the $25 \mathrm{x}$ lens was used for IpDip, too. The slicing was kept constant, although the use of IpS in conjunction with the $25 \mathrm{x}$ lens would enable a larger slicing. Therefore, and since we choose a small writing scan field $\left(150 \times 150 \mu \mathrm{m}^{2}\right)$, the larger scanning field of the $25 \mathrm{x}$ lens in comparison to the $63 \mathrm{x}$ lens did not lead to high throughput. All lenses (Figures $5 \mathrm{a}-\mathrm{c}$ ) were written with $34 \mathrm{~s}$ writing time per single lens or about 5 min for the $3 \times 3$ MLA. The comparison shows that an increased voxel overlap reduces the roughness significantly (Figure $5 \mathrm{a}$ vs. $5 \mathrm{~b}$ ). The resist blurring due to the changed resist is also clearly visible in the central part of the lens (Figure 5b vs. 5c).

Further improvements in the surface roughness can be achieved by reduction of the slicing distance. First, we show the reduction from $500 \mathrm{~nm}$ to $300 \mathrm{~nm}$, for which still some details of layering could be seen in the deep sags of the concave lenses (Figure 5c vs. 5d). Finally, for $50 \mathrm{~nm}$ slicing almost no roughness could be seen in the SEM micrograph from layering but some material intrinsic and granular surface roughness remains (Figure 5e). However, the micrograph displaying the lens which was obtained using smoothening by selective thermal reflow is even less rough and shows a superior surface finish (Figure 5f). Although roughness values were not quantitatively analyzed using scanning force microcopy on the microlenses, both values displayed in Figure 5e and Figure $5 \mathrm{f}$ are well below $10 \mathrm{~nm}$. This goes along with the results by other researchers in Ref. [12], where $10 \mathrm{~nm}$ roughness was obtained with $150 \mathrm{~nm}$ slicing with IpS in the solid writing mode. However, the writing time of the structure in IpS with $50 \mathrm{~nm}$ slicing is 10 times or one order of magnitude larger than that of the original structure in IpDip. Such differences in writing times will result in huge processing times, e.g., if lens arrays with a few millimeters of diameter need to be exposed. As an example, for a lens structure with $2.4 \mathrm{~mm}$ diameter, consisting of about 1'800 lenses with $50 \times 50 \mu \mathrm{m}^{2}$ footprint each, the exposure time is already 48 hours, even for the $500 \mathrm{~nm}$ slicing, including significant overhead times 


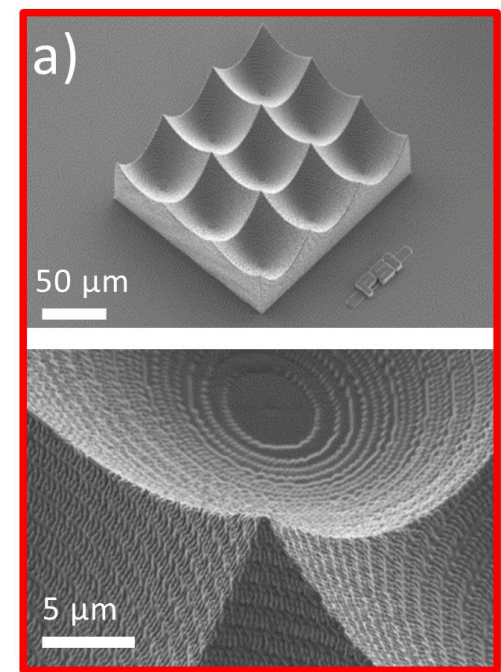

63x, IpDip, slicing $500 \mathrm{~nm}$
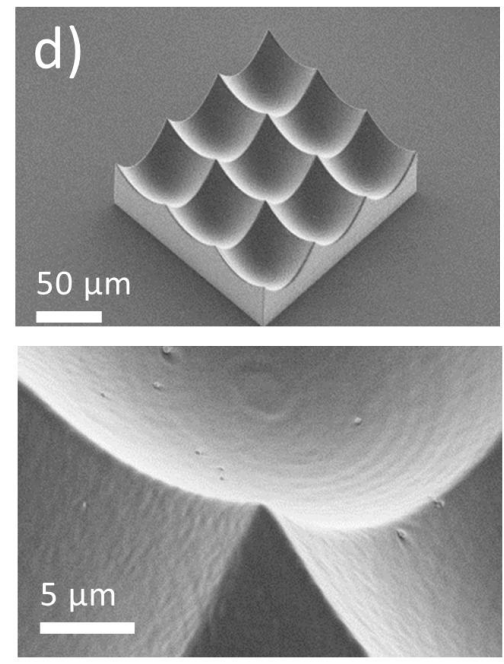

25x, IpS, slicing $300 \mathrm{~nm}$
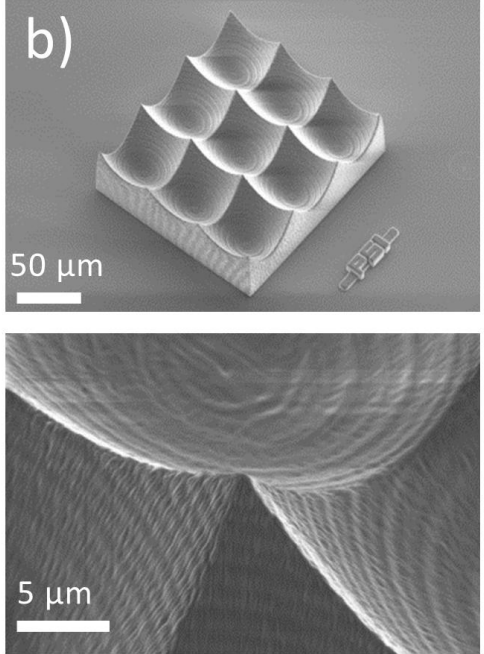

25x, IpDip, slicing $500 \mathrm{~nm}$
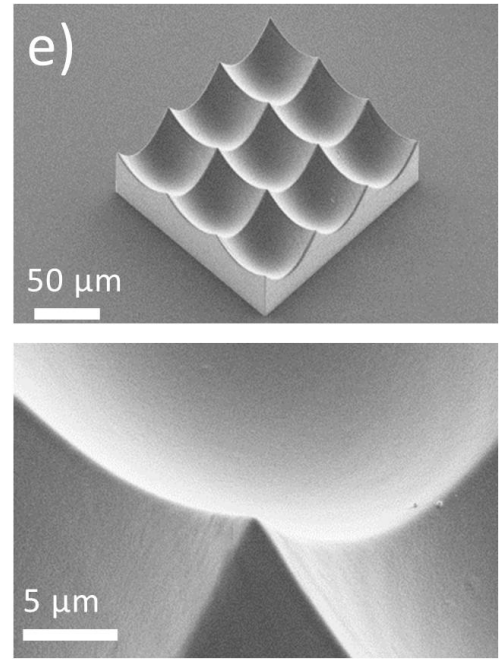

25x, IpS, slicing $50 \mathrm{~nm}$
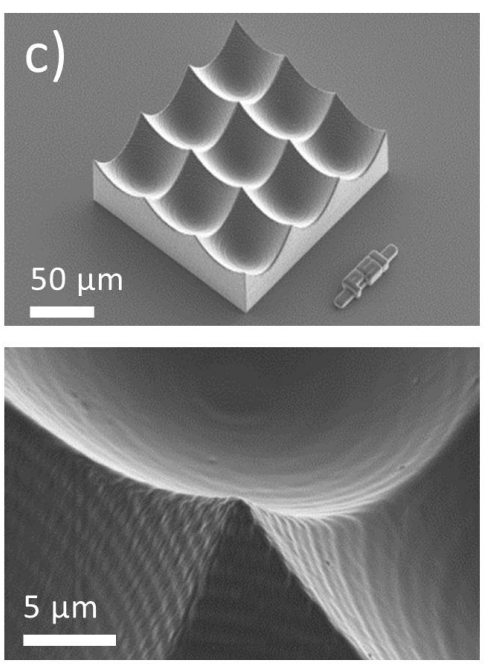

25x, IpS, slicing $500 \mathrm{~nm}$

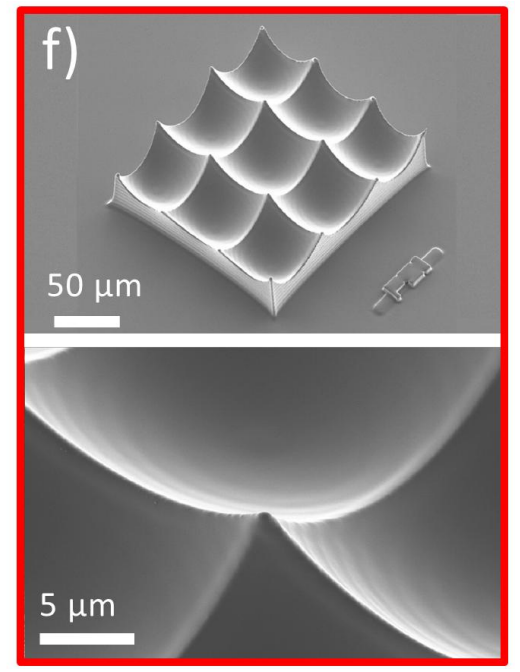

63x, IpDip, slicing $500 \mathrm{~nm}$, reflow

Figure 5. SEM micrographs of microlens array manufactured by 2PP-DLW displaying the effect of variation of voxel size (resist with "intrinsic diffusion", magnification of microscope objective), writing strategy (slicing) and post processing (TASTE) on surface roughness. Upper row: $3 \times 3$ lens array, lower row: magnification of intersection of 4 lenses. a-c) with different microscope lens and resist combinations but slicing distance $500 \mathrm{~nm}$ constant (resulting in 5 min writing time): a) with $63 x$ in IpDip, b) with $25 x$ in IpDip, c) with $25 x$ in IpS. d)-e) with different slicing distances both with $25 x$ in IpS: d) $300 \mathrm{~nm}$ (in $8.5 \mathrm{~min}$ writing time), e) $50 \mathrm{~nm}$ (in 50 min writing time). f) For comparison with post processing using selective thermal reflow with lenses from a) with $63 x$ in IpDip, indicated by red frames.

Small differences, e.g., the tip at the intersection of the 4 lenses and the right slope of the surface smoothened structure in Figure $5 \mathrm{f}$ indicate some minor issues. Although the structures are in principle prone to collapse, this was not observed for the surface smoothening. For the concave structures with highly varying slopes shown here, the surface smoothening seems to work over steps of up to $400-500 \mathrm{~nm}$, from the lens bottom with the shallow ring sections up to the sharp tips with the $<1 \mu \mathrm{m}$ radius. One can argue that roughness reduction by surface reflow is physically different from the writing with enlarged blurred voxel. In comparison to the enhanced overlapping of voxels, which leads to a merging of cylinderlike trajectories into a continuous surface, it is based on material displacement from elevated areas into small depressions, and thus is a true equilibration process which minimizes the energy [23,24]. However, one should not forget that the damage of the PMMA during exposure results in a surface skin with low molecular weight, which has to be taken into account for further process steps involving temperature treatment or aggressive solvents. 


\section{CONCLUSION}

The aim of this publication was to demonstrate the efforts necessary to achieve similar surface roughness by reducing the slicing distance as with smoothening by post-processing. While the reduction of the slicing distance, including strategies to write structural shells with smaller increment than the inner part or even a scaffolding of the lens is the way to go if high throughput structures with resolution elements have to be directly written, the post-processing is a way to achieve surface roughness from structures which were optimized for speed and not surface roughness during origination with 2PP-DLW. Due to the lack of availability of production machines for 2PP-DLW (apart from the newer developments presented by Multiphoton Optics $\mathrm{GmbH}$ ), throughput will remain an issue for the fabrication of extended arrays of lenses. Scaling larger arrays and higher structures will critically enhance the writing time and the post-processing will remain in this area as a valuable and flexible method to obtain smooth surfaces Since in the future there will be both a need for original structures and such being used as originals for replication, different strategies need to be developed. The NIL process chain with its main elements origination, replication and pattern transfer (functionalization), is a good example to demonstrate this capability [25,26].

While the process is here related to surface roughness leading to structural defects, diffraction and scattering, the aim is to show how lithography and the locally selective modification of material properties cannot only enhance the surface quality of micro-optical elements, but eventually speed up the manufacturing to allow the manufacturing of lens arrays within times acceptable for today's 2PP-DWL tools. The trade-off is that two additional replication steps are needed. Once new materials are found which allow both 2PP-DWL and post-processing, this will be greatly facilitated.

\section{ACKNOWLEDGEMENT}

This project was funded by the Swiss Nanoscience Institute (project A10.13 SurfFlow). The authors express their special thanks to K. Vogelsang (PSI) for support with the hot embossing and M. Vockenhuber (PSI). The authors also thank M. Rossi (Heptagon) for the support of this project and the useful discussion.

\section{REFERENCES}

[1] Kirchner, R., Chidambaram, N., Altana, M., and Schift, H., "Reducing the roughness of 3D micro-optics," 3 January 2017, SPIE Newsroom (Micro/Nano Lithography).

[2] Gale, M.T., [Replication], in Micro-optics, H.P. Herzig, ed., Taylor \& Francis, London, 153-178 (1997).

[3] Gale, M.T., Rossi, M., Schuetz, H., "Fabrication of continuous-relief micro-optical elements by direct laser writing in photoresist, laser-assisted fabrication of thin films and microstructures," Proc. SPIE 2045, 54-62 (1994).

[4] Kirchner, R., Guzenko, V. A., Vartiainen, I.; Chidambaram, N. and Schift, H., "ZEP520A - A resist for electron-beam grayscale lithography and thermal reflow," Microelectron. Eng. 153, 71-76 (2016).

[5] Kirchner, R., Guzenko, V. A., Rohn, M., Sonntag, E., Mühlberger, M., Bergmair, I. and Schift, H., "Bioinspired 3D funnel structures made by grayscale electron-beam patterning and selective topography equilibration", Microelectron. Eng. 141, 107-111 (2015).

[6] Stavis, S. M., Strychalski, E. A. and Gaitan, M., "Nanofluidic structures with complex three-dimensional surfaces," Nanotechnology 20, 165302 (7 pp) (2009).

[7] Keskinbora, K., Grévent, C., Hirscher, M., Weigand, M. and Schütz, G., "Single-Step 3D Nanofabrication of Kinoform Optics via Gray-Scale Focused Ion Beam Lithography for Efficient X-Ray Focusing," Adv. Opt. Mater. 3, 792-800 (2015).

[8] Maruo, S., Nakamura O. and Kawata S., "Three-dimensional microfabrication with two-photon-absorbed photopolymerization," Opt. Lett. 22(2), 132-134 (1997).

[9] Harnisch, E., Russew, M., Klein, J., König, N, Crailsheim, H. and Schmitt, R., "Optimization of hybrid polymer materials for $2 \mathrm{PP}$ and fabrication of individually designed hybrid microoptical elements thereof," Opt. Mat. Expr. 5(2), 456-461 (2015).

[10] Chidambaram, N., Kirchner, R., Altana, M., and Schift, H., "High fidelity 3D thermal nanoimprint with UV curable polydimethyl siloxane stamps," J. Vac. Sci. Technol. B 34(6) 06K401 (2016). 
[11] Dietrich, P.-I., Reuter, I., Blaicher, M., Schneider, S., Billah, M. R., Hoose, T., Hofmann, A., Caer, C., Dangel, R., Offrein, B., Moehrle, M., Troppenz, U., Zander, M., Freude, W., Koos, C., "Lenses for low-loss chip-tofiber and fiber-to-fiber coupling fabricated by 3D direct-write lithography," Conf. on Lasers and Electro-Optics (CLEO’16), San Jose (CA), USA, June 5 - 10 , paper SM1G.4. (2016).

[12] Gissibl, T., Thiele, S., Herkommer, A., and Giessen, H., "Two-photon direct laser writing of ultracompact multi-lens objectives," Nature Photonics 10, 554-560 (2016).

[13] Eckhardt Optics LLC, online, accessed 2017-01-23, http://eckop.com/optical-scatter-2/optical-scattering-versussurface-roughness/

[14] Bennett, H. E. and Porteus, J. O., "Relation between surface roughness and specular refectance at normal incidence,” J. Opt. Soc. of Am., 51:123\{129, 1961.

[15] Takada, K., Sun, H.B., and Kawata, S, "Improved spatial resolution and surface roughness in photopolymerization-based laser nanowriting," Applied Physics Letters 86, 071122, (2005).

[16] Stuart, B.C., Feit, M.D., Herman, S., Rubenchik, A.M., Shore, B.W., and Perry, M.D., "Nanosecond-tofemtosecond laser-induced breakdown in dielectrics," Physical Review B 53(4), 1749 (1996).

[17] Guo, R., Xiao, S., Zhai, X., Li, J., Xia, A., and Huang, W., "Micro lens fabrication by means of femtosecond two photon photopolymerization," Optics Express 14, 810-816 (2006).

[18] Wu, D., Wu, S.Z., Niu, L.G., Chen, Q.D., Wang, R., Song, J. F., Fang, H.H., and Sun, H.B., "High numerical aperture microlens arrays of close packing," Applied Physics Letters 97, 031109 (2010).

[19] Schleunitz, A., Guzenko, V. A., Messerschmidt, M., Atasoy, H., Kirchner, R., and Schift, H., "Novel 3D microand nanofabrication method using thermally activated selective topography equilibration (TASTE) of polymers," Nano Convergence 1,7 (2014).

[20] Chidambaram, N., Kirchner, R. Fallica, R. Yu, L. Altana, M., and Schift H., "Selective surface smoothening of polymer microlenses by depth confined softening," submitted (2017).

[21] Schift, H., Spreu, C., Saidani, M., Bednarzik, M., Gobrecht, J.. Klukowska, A., Reuther, F., Gruetzner, G. and Solak, H.H., "Transparent hybrid polymer stamp copies with sub-50 nm resolution for thermal and UVnanoimprint lithography," J. Vac. Sci. Technol. B 27(6), 2846-2849 (2009).

[22] Schift, H., "Nanoimprint lithography: an old story in modern times? A review," J. Vac. Sci. Technol. B 26(2), 458-480 (2008).

[23] Kirchner, R., Schleunitz, A., and Schift, H., "Energy-based thermal reflow simulation for 3D polymer shape prediction using the surface evolver," J. Micromech. Microeng. 24(5), 055010 (7pp) (2014)

[24] Kirchner, R. and Schift, H., "Mobility based 3D simulation of selective, viscoelastic polymer reflow using Surface Evolver," J. Vac. Sci. Technol. 32(6), 06F701 (7pp.) (2014).

[25] Schift, H., Urwyler, P., Kristiansen, P.M., and Gobrecht, J., "Nanoimprint lithography process chains for the fabrication of micro- and nanodevices," J. Micro/Nanolith. MEMS MOEMS 13(3), 031303 (2014).

[26] Schift, H., "Nanoimprint lithography, 2D or not 2D? A review," Appl. Phys. A 21(2), 415-435 (2015). 